\title{
GASTROINTESTINAL ULTRASONOGRAPHY OF THE DOG: A REVIEW OF 265 CASES (1996-1998)
}

\author{
F. MANCZUR ${ }^{*}$ and K. VÖRÖS \\ Department and Clinic of Internal Medicine, University of Veterinary Science, \\ H-1400 Budapest, P.O. Box 2, Hungary
}

(Received July 18, 1999; accepted October 20, 1999)

\begin{abstract}
The findings of ultrasonography of the gastrointestinal (GI) tract of 265 dogs with GI disorders were analysed retrospectively. The sonographic changes associated with various inflammatory and neoplastic conditions and mechanical obstruction of the GI system were recorded and discussed. Sonographic alterations of the pancreas and the tissues adjacent to the GI tract were also included in the study. Ultrasonographic alterations of the GI tract were classified into three main categories: thickening of the GI wall, changes in peristalsis and dilation of the lumen. Localised thickening of the GI wall with disruption of its structure was caused by both neoplastic diseases and by inflammatory disorders. However, diffuse thickening with retained wall structure was generally associated with inflammatory diseases. The criteria previously established for the ultrasonographic diagnosis of intestinal obstruction were successfully applied to a large number of GI disorders. Pancreatitis was most often associated with hyperechoic mesentery and hypoechoic pancreas mass, but similar alterations were encountered in some cases of gastric or duodenal ulceration. Except in cases of invaginations and intestinal obstructions, the observed ultrasonographic changes were not specific enough for a definitive diagnosis. Nevertheless, ultrasonography proved to be a valuable technique in the diagnostic process of GI disorders of the dog.
\end{abstract}

Key words: Ultrasonography, dog, gastrointestinal diseases, pancreas

Ultrasonography is increasingly used during the diagnosis of various ga strointestinal (GI) disorders both in human and veterinary medicine (Mittelstaedt, 1992; Penninck, 1995; Lamb, 1999). The normal ultrasonographic appearance and wall thickness of the intestinal tract of the dog have been described ( Penninck et al., 1989). Changes in the thickness and appearance of the GI wall, in the diameter and content of the GI lumen and in the peristalsis can all be assoc iated with pathological processes (Penninck et al., 1990).

The objectives of this retrospective study were to assess the diagnostic value of the observed ultrasonographic alterations on a large number of clinical cases and

*E-mail: fmanczur@univet.hu; Fax: +36 (1) 4784137 
to combine our findings with those of earlier reports in order to determine the role of ultrasonography in the diagnosis of canine gastroenterological diseases.

\section{Materials and methods}

Data of dogs with possible signs of gastrointestinal disorder that were e $\mathrm{x}$ amined ultrasonographically by the same author (FM) between May 11996 and April 301998 were analysed. The indications for ultrasonography were vomiting, chronic diarrhoea, abdominal pain, rectal bleeding, palpable mass in the abdomen and chronic weight loss. Dogs were examined with ultrasound scanners equipped with a $3.5-5 \mathrm{MHz}$ convex array and a $7 \mathrm{MHz}$ sector transducer (Brüel and Kjaer 1846 and Brüel \& Kjaer Panther 2002, Naerum, Denmark).

The abdominal skin was prepared as for a routine abdominal ultrasound e $\mathrm{x}$ amination (clipping of the hair, wetting with ultrasound gel). All ultrasonographic examinations were performed on conscious dogs in dorsal and right lateral recu mbency. When the pylorus and the proximal part of the duodenum could not be identified using this approach, the dogs were positioned oblique between dorsal and right lateral recumbency. When gas-containing parts of the gastrointestinal tract interfered with the transmission of ultrasound, scanning from the dependent side of abdomen was tried. Often a slight increase of pressure with the transducer was used to displace superficial, gas-containing intestinal loops.

\section{Results}

Out of the approximately 300 examined dogs, those 265 were selected for this study where the final diagnosis could be established on the basis of postmortem examination, histological examination, result of surgery or the clinical outcome of the cases. The population included 154 males and 111 females. Fiftythree dogs were of mixed breed, while the other 212 belonged to 52 different ped igree breeds. Their age ranged from 2 months to 16 years ( mean: 5.1 years).

The animals were classified into 8 groups according to their final $\mathrm{d}$ iagnosis.

\section{Inflammatory conditions}

Altogether 163 dogs had GI symptoms caused by various primary (Fig. 1) or secondary inflammatory disorders (e.g. those resulting from uraemia) of some segments of the GI tract (excluding the pancreas), maldigestion, constipation, ph aryngeal or oesophageal foreign body, megaoesophagus, drug-induced or posto perative motility disorder or non-gastrointestinal disease (e.g. pyometra, septica emia). The ultrasonographic fin dings are listed in Table 1. 


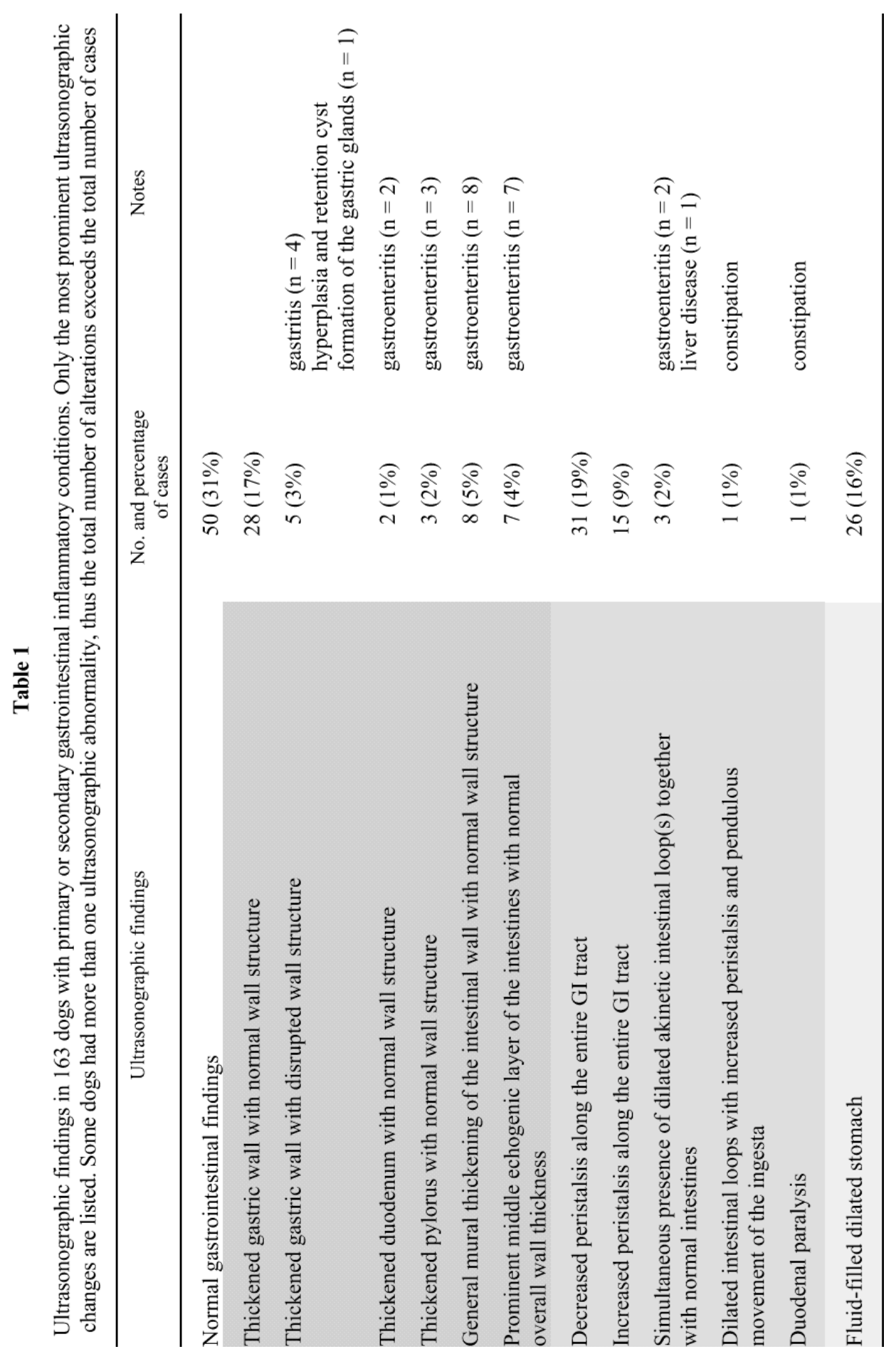

Acta Veterinaria Hungarica 48, 2000 


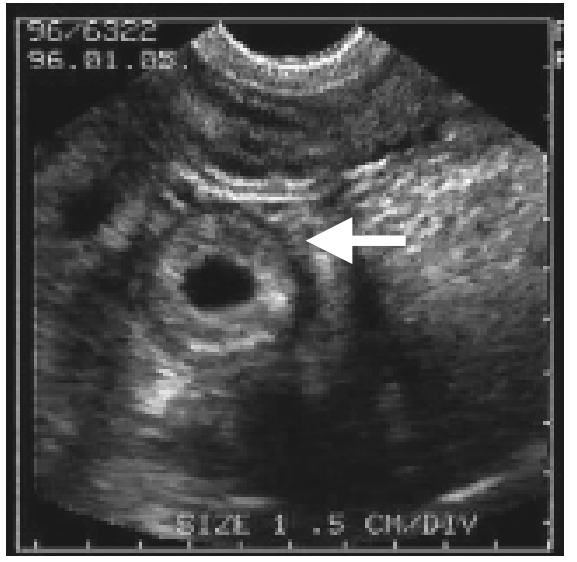

Fig. 1. Ultrasonographic image of a small intestinal loop of a dog in transverse section (arrow).

The intestinal wall is thickened with retained structure. The histological diagnosis revealed lymphocytic-plasmacytic enteritis

\section{Congenital anomalies}

There were 2 dogs with congenital diseases of the GI tract (one dog with congenital hypertrophic pyloric stenosis and another one with idiopathic muscular hyperplasia of the small intestines). Ultrasonography revealed thickened wall with normal layered structure of the affected GI segments (pylorus or small intestines) in both cases. Thickening of the outer hypoechoic layer of the small intestinal wall was obvious in the dog with muscular hyperplasia (Fig. 2).

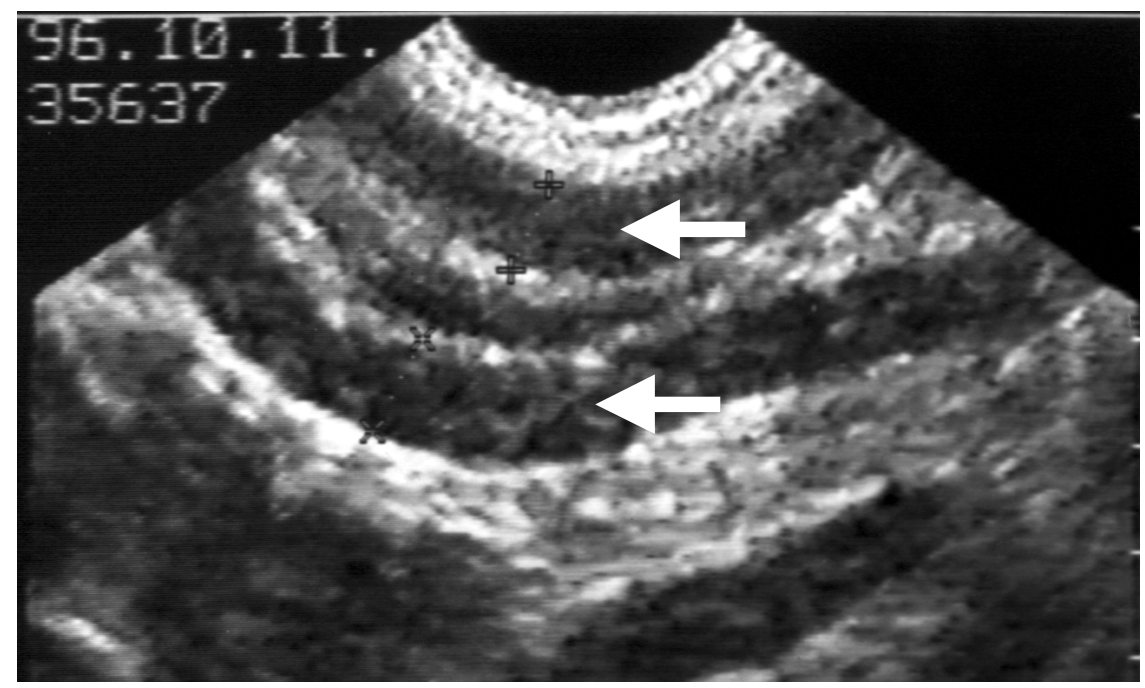

Fig. 2. Ultrasonographic image of the duodenum of a dog with idiopathic muscular hypertrophy of the entire small intestinal tract. The duodenal wall is thickened and seems hypoechoic because of the prominent muscular layer. A thin hyperechoic line in the middle of the wall represents the submucosa (arrows) 


\section{Gastric foreign bodies}

Non-obstructive gastric foreign bodies were diagnosed in two dogs. Ech ogenic reflex with shadowing and thickened gastric wall was found by ultrasound in one of them, however no abnormality was o bserved in the other case.

\section{Intestinal obstruction}

Mechanical obstruction of the GI tract was diagnosed in 33 dogs including 14 dogs with intestinal invaginations, 10 dogs with ingested foreign bodies, 5 dogs with i ntestinal adhesions and one case each of intestinal torsion, linear foreign body (Fig. 3), diaphragmatic hernia and partial obstruction caused by intestinal tumour (liposa rcoma).

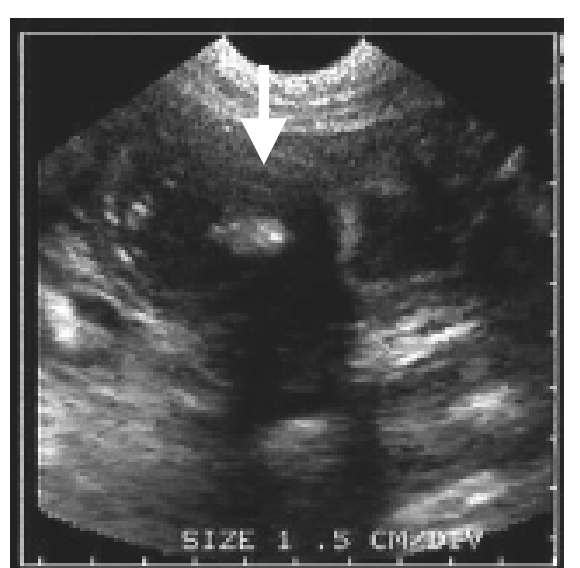

Fig. 3. Ultrasonographic image of the intestines of a dog with linear foreign body. The intestines are dilated and have plicated appearance. The foreign body is visible as a hyperechoic reflex with shadowing in the middle of the intestinal lumen (arrow)

Invaginated intestines were detected by ultrasound in 13 of the 14 dogs with intussusceptions (93\%); however, in two of these dogs no invaginated intestines were found during surgery. The observed sonographic changes are listed in Table 2.

\section{Gastrointestinal neoplasia}

Besides the dog with liposarcoma that caused partial obstruction, which was thus included in the intestinal obstruction cases, further 8 dogs were diagnosed as having GI neoplastic processes. There were six dogs with GI lymphoma (restricted to some intestinal loops in five dogs and involving both the stomach and the inte stines in one case) and one dog with rectal and another one with gastric polyps. All neoplastic alterations appeared as focal hypoechoic mural masses of the affected GI segments during ultrasonography (Fig. 4). Enlarged regional lymph nodes were found by ultrasound in 4 cases, but they were misinterpreted as cystic pancreatic 1 esions in one dog. Hyperechoic mesenteric fat around the affected intestine was noted in one dog with intestinal lymphoma. In one dog, ultrasonography was normal 2.5 months prior to detecting intestinal lymphoma by a repeated ultrasound exam ination. 


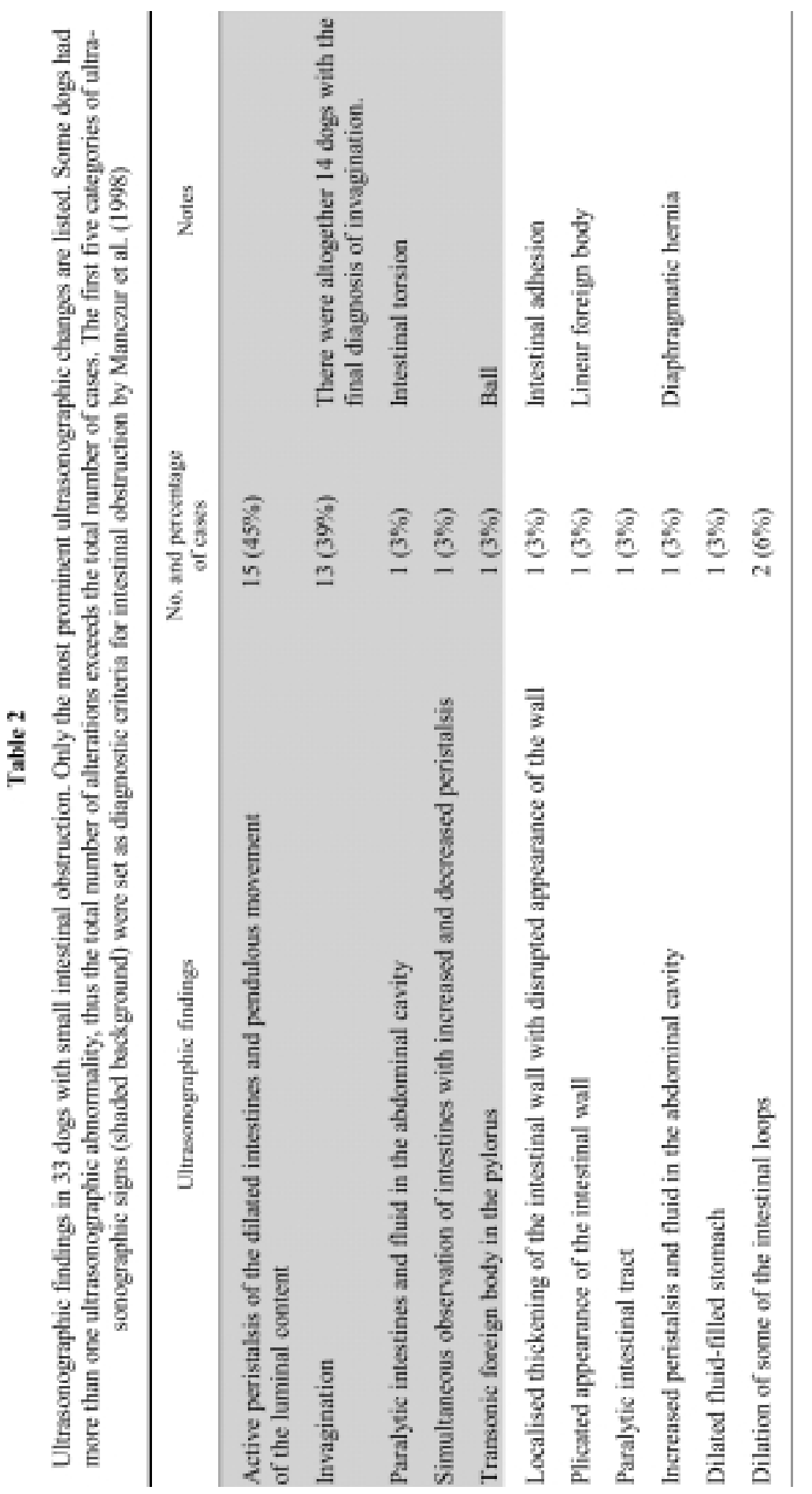




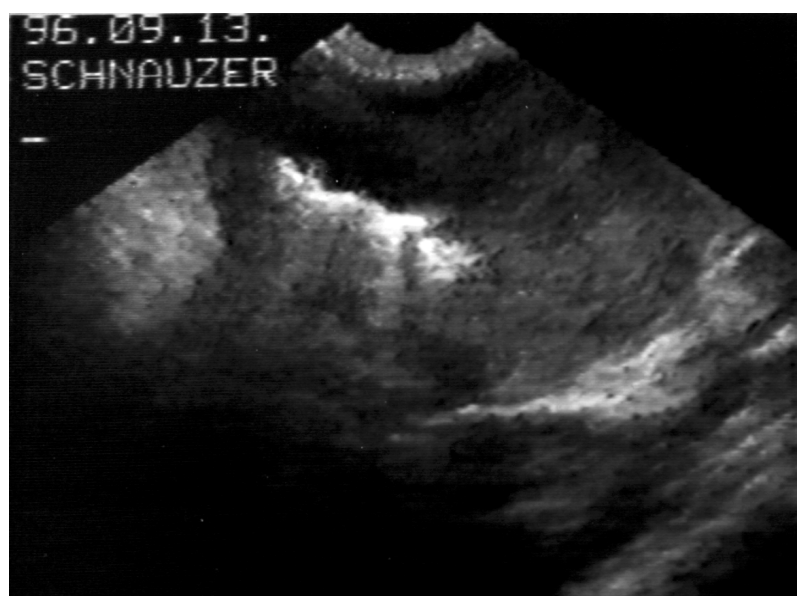

Fig. 4. Ultrasonographic image of intestinal lymphoma. The intestinal wall is markedly thickened and homogeneously hypoechoic without recognisable layers. Gas artefact in the intestinal lumen helps to locate the origin of the mass to the GI tract

\section{Gastrointestinal ulceration}

Gastrointestinal ulceration was diagnosed in 9 dogs. Ultrasonography $\mathrm{r}$ evealed mural thickening with retained wall structure of the stomach in 6 cases, that of the duodenum in 2 cases and that of the pylorus or the small intestines in one case each. Hyperechoic mesentery was noted in 4 dogs, and hypoechoic lesions in the pancreas region were found in 2 dogs. Two dogs had thickened gastric wall with disrupted structure. Dilated, fluid-filled stomach was observed in two p atients, and in one of them this was the only observed ultrason ographic alteration.

\section{Gastrointestinal perforation}

Two dogs were diagnosed with intestinal perforation. Ultrasonography d etected paralytic ileus in both cases, while dilated stomach, irritated appearance of the duodenum and free abdominal fluid appeared in one of them.

\section{Diseases of the pancreas}

There were 43 dogs with acute pancreatitis and 3 dogs had pancreatic $t \mathrm{u}-$ mour (two had adenocarcinoma and one dog demonstrated the clinical signs of a possible insulinoma). Ultrasonography revealed hypoechoic pancreas masses and hyperechoic peripancreatic mesentery in $33(77 \%)$ and in $29(67 \%)$ out of the 43 dogs with pancreatitis, respectively. The pancreas seemed hyperechoic in one case (2\%) and had mixed echogenicity in 3 cases $(7 \%)$ of pancreatitis. Pancreatitis caused wall thickening with normal structure of the stomach in 7 dogs $(16 \%)$, that of the pylorus in 2 dogs $(5 \%)$, that of the duodenum in 10 dogs $(23 \%)$ and that of 
the small intestines in one case (2\%). The normal layered appearance of the thic kened gastric wall was lost in one animal with pancreatitis (2\%). Other ultrason ographic alterations associated with pancreatitis were dilated stomach in 4 dogs (9\%), duodenal paralysis in 3 dogs $(7 \%)$, and irritated appearance of the duodenum in 2 dogs $(5 \%)$. No ultrasonographic alterations in the pancreatic region were found during three repeated examinations of a dog with the clinical signs of insul inoma. Ultrasonography detected hyperechoic peripancreatic mesentery, dilated stomach, thickened gastric wall with normal structure, and duodenal paralysis in the two dogs with pancreatic adenocarcinoma.

\section{Discussion}

The ultrasonographic changes of different GI diseases can be classified into three main categories:

1. Localised or diffuse thickening of the GI wall,

2. Changes in peristaltic activity,

3. Dilatation of the lumen.

\section{Thickening of the GI wall}

Pathological thickening of the GI wall of the dog should be suspected when the wall of the stomach measures more than $6-7 \mathrm{~mm}$ and when the intestinal wall measures more than $5 \mathrm{~mm}$ (Penninck et al., 1989). Localised thickening of the GI wall together with disruption of the layered appearance may be observed in different neoplastic processes, in severe inflammatory disease, in pancreatitis or in GI ulcers (Penninck et al., 1990; Saunders, 1991; Myers and Penninck, 1994; KaserHotz et al., 1996; Penninck et al., 1997; Rivers et al., 1997). Thickened gastric wall with irregularly layered appearance (i.e. pseudolayering) is reported to occur in gastric epithelial tumours (Penninck et al., 1998). Observation of regional lymph node enlargement will further increase the likelihood of a neoplastic process a ccording to Kaser-Hotz et al. (1996) and Penninck et al. (1998). Diagnosis can only be achieved by percutaneous or intraoperative biopsy techniques where the former is being performed under ultrasound guidance (Crystal et al., 1993; Penninck et al., 1993). Localised thickening of one layer of the GI wall can be seen in congenital pyloric stenosis or in chronic hypertrophic pyloric gastropathy ( Penninck et al., 1990; Biller et al., 1994). Pathological enlargement of the pylorus should be co nsidered in small- to medium-sized dogs if the length of the pyloric canal exceeds $20 \mathrm{~mm}$, the muscular layer and the pyloric $\mathrm{w}$ all is thicker than $4 \mathrm{~mm}$ and $8-9 \mathrm{~mm}$, respectively, while the overall diameter of the pylorus is more than $26 \mathrm{~mm}$ (Penninck et al., 1990; Biller et al., 1994; Agut et al., 1996). Localised thickening with too many wall layers is observed in cases of intestinal invaginations ( Flückiger and Arnold, 1986; Kantrowitz et al., 1988; Penninck et al., 1990; Watson et al., 1991). 
The 'intestine in intestine' or 'target pattern' of intussusceptions is one of the most easily recognisable GI abnormalities. Diffuse thickening with normal layered a ppearance generally indicates inflammatory disease or pancreatitis, but diffuse ne oplastic disease should also be included in the differential diagnosis ( Penninck et al., 1990; Grooters et al., 1994; Penninck, 1995; Spohr et al., 1995). Some cases of enteritis may not cause detectable structural changes of the intestinal tract or may result in only luminal distension without wall thickening. Again, diagnosis can only be achieved by histological examination of biopsy specimens.

The findings of the present study were in accordance with previous $r$ eports.

Localised thickening of the GI wall with disrupted structure were detected by ultrasonography in 8 dogs with neoplastic disease and in 10 dogs with infla mmatory disorders.

Diffuse thickening with retained wall structure seen in 56 dogs, however, was always associated with inflammatory disease in our cases. To our know 1edge, the ultrasonographic appearances of muscular hypertrophy of the small i ntestines and gastric glandular hyperplasia described in the present study have not been published earlier.

Prominent middle echogenic layer of small intestines with normal overall wall thickness was noted in 7 dogs with gastroenteritis. This layer corresponds to the submucosa plus interface echoes with the adjacent layers and it normally a ppears as thick as, or thinner than, the mucosa on the sonograms. The observed prominent appearance of submucosa in these cases may be explained by cellular infiltration of this layer and/or by villous atrophy that caused the thinning of the mucosal layer. Unfortunately, no histological samples were available from any of these cases, thus the exact explanation of this ultrasonographic sign needs to be sought further.

As in earlier studies, invaginations were detected with high accuracy also in this study. In two dogs, no invaginated intestines were found during surgery fo 1lowing the ultrasonographic diagnosis of this disorder. Because the ultrason ographic alterations are very specific for intestinal invaginations, the most likely e $\mathrm{x}$ planation is that the invaginations had spontaneously resolved by the time of su rgery. Whether or not anaesthesia played a role in this process needs to be invest igated further. Detection of blood flow in the wall of invaginated intestinal se gments using colour Doppler ultrasonography is used to determine the viability of intestines, and thus to decide further treatment (conservative versus surgery) in children (Lagalla et al., 1994). Using a similar approach in canine patients may help to judge whether or not immediate surgery is needed. However, the risks of delaying surgery in cases that do not resolve spontaneously or following conserv ative treatment (enema) should be weighed against those cases where unnecessary operations can be avoided. 


\section{Changes in peristaltic activity}

A general increase in GI peristalsis with uniform luminal content movement and mild dilatation of the GI lumen was reported in inflammatory disease ( Manczur et al., 1998). Observation of a localised decrease or lack of peristalsis may be the result of acute pancreatitis and severe localised inflammatory or neoplastic di seases (Penninck et al., 1990; Myers and Penninck, 1994; Nyland et al., 1995; Rivers et al., 1997). Generalised decrease or absence of peristalsis with dilated lumen can result from severe inflammatory disease, gastric ulceration, prolonged $\mathrm{m} \mathrm{e}$ chanical obstruction, severe systemic disease or administration of certain drugs (Penninck et al., 1990; Tidwell and Penninck, 1992; Myers and Penninck, 1994; Penninck et al., 1997; Manczur et al., 1998). Increased peristaltic activity with pendulous movement of the ingest inside the dilated intestinal loop is a sensitive sign of mechanical obstruction (Manczur et al., 1998). Simultaneous observation of different intestinal loops with both increased and decreased peristaltic activity also suggests possible mechanical o bstruction (Manczur et al., 1998).

The findings obtained on peristalsis in the present study were similar to the results reported in the above-mentioned publications.

Normal, generally increased or decreased peristalsis was associated with non-obstructive disorders in 237 out of our 239 cases. Paralytic ileus was found in a dog with prolonged mechanical obstruction, and increased peristalsis was noted in a dog with diaphragmatic hernia.

Pendulous movement of the ingesta caused by active peristaltic contractions of the dilated intestine was a sensitive sign of intestinal obstruction. It was assoc iated with mechanical ileus in 15 of the 16 observed cases.

Localised paralysis of the intestines, however, is not a sensitive sign of intestinal obstruction, because this finding was associated with non-obstructive di seases in 7 out of the 9 observed cases.

\section{Dilatation of the lumen}

Dilatation of the stomach was observed postprandially, or as a result of a primary or secondary gastric emptying problem. In case of severe dilatation, the authors sometimes observed compartmentalisation of the gastric lumen during and outside the study period. This warrants further investigations (e.g. radiography) to exclude the possibility of gastric to rsion.

Mild dilatation of the intestinal lumen was seen together with normal peristaltic activity of the gut or in inflammatory disorders.

In contrast, marked dilatation of the lumen was observed in paralytic or mechanical ileus cases, as also described earlier by Penninck et al. (1990) and Manczur et al. (1998). Generally, more severe dilatation of the intestines was noted in mechanical than in paralytic ileus, but sonographic measurement of the luminal d iameter of the dilated intestines was not pe rformed in the present study. 
Large amount of intraluminal gas was present in the intestines of dogs with digestive disorders, or as the effect of intestinal obstruction. Besides accumulation of large amounts of intraluminal gas and fluid, intestinal strangulation (volvulus) also produced rapid accumulation of free abdominal fluid ( Manczur et al., 1998). Large amount of intraluminal gas was often observed in dogs without GI disorders by the authors outside of the present study period.

Gastrointestinal foreign bodies can sometimes be detected by ultrasonogr aphy in the stomach or intestinal lumen. Their sonographic picture depends on their shape and acoustic properties (Penninck et al., 1990; Tidwell and Penninck, 1992). They often cause shadowing that may be differentiated from that caused by gas, based on the lack of internal echoes inside the shadow ('clean' shadow). In co ntrast, gas generally produces 'dirty' shadowing but the differentiation between the two is not always possible (Tidwell and Penninck, 1992; Manczur et al., 1998). Linear foreign bodies can appear as echogenic thin intraluminal objects with or without acoustic shadowing. The intestines also look 'plicated' in some cases of linear foreign bodies (Tidwell and Penninck, 1992).

The presence of intraluminal foreign bodies was observed in only 3 out of the 13 cases of this study. Nevertheless, changes in peristaltic activity or in the a ppearance of the GI wall (plication, thickening), or dilation of the lumen were noted in all but one dog with a gastric foreign body.

Because of the functional and anatomical relationship of the pancreas to the GI tract, the findings of the examination of that organ were also included in this study. Like previous reports, we also found that visualisation of the normal canine pancreas was difficult or even impossible because of its similar echogenicity to the surrounding tissues (Saunders, 1991; Lamb and Simpson, 1995; Nyland et al., 1995). Therefore, instead of visualisation of the pancreas itself, thorough scanning of the pancreatic region was performed. A negative sonographic finding probably does not exclude the possibility of a milder disease of the organ, but in the absence of histological examinations, the accurate interpretation of negative ultrason ographic findings is difficult, because neither plain film radiography nor blood chemistry is sensitive and specific enough for a definitive diagnosis. In 37 out of the 40 cases when the pancreas was easily recognisable in this study, this was a ssociated with acute or subacute pancreatitis. Generally echo-poor lesions or masses were found in a more or less hyperechoic peripancreatic mesentery, but increased or mixed echogenicity of the pancreas was also noted. Thickened duodenal or ga stric wall, paralytic duodenum, irritated appearance of the adjacent intestines, $\mathrm{d}$ ilated common bile duct and hepatic lesions often accompanied the structural changes within the pancreas. These changes have already been described in detail by Saunders (1991) and by Nyland et al. (1995). The sonographic alterations were not specific enough to distinguish among the different types of pancreatitis and, interestingly, only secondary changes of the surrounding tissues were observed in the two dogs with histologically proven pancreatic tumour. Furthermore, the above- 
mentioned ultrasonographic features were also noticed in other gastrointestinal di seases. For example, hyperechoic peripancreatic mesentery and/or hypoechoic lesions in the pancreas region were also seen in some cases of gastric or duodenal ulcer ation.

Based on the results of this retrospective study it can be concluded that $\mathrm{u} l-$ trasonography is a useful diagnostic imaging method in GI abnormalities of the dog. Changes in GI wall structure, peristalsis, luminal diameter and content, and morphological alterations of the pancreas and adjacent organs can be readily $\mathrm{d}$ etected by this imaging modality. Even though the observed changes (except i nvagination and mechanical obstruction) are not specific enough for a definitive $d$ iagnosis, ultrasonography is of value in facilitating decision-making on further $\mathrm{d} i$ agnostic processes or treatment of GI diseases of the dog.

\section{References}

Agut, A., Wood, A. K. W. and Martin, I. C. A. (1996): Sonographic observations of the gastroduodenal junction of dogs. Am. J. Vet. Res. 57, 1266-1273.

Biller, D. S., Partington, B. P., Miyabayashi, T. and Leveille, R. (1994): Ultrasonographic appearance of chronic hypertrophic pyloric gastropathy in the dog. Vet. Radiol. and Ultrasound 35, 30-33.

Crystal, M. A., Penninck, D. G., Matz, M. E., Pearson, S. H., Freden, G. O. and Jakowski, R. M. (1993): Use of ultrasound-guided fine-needle aspiration biopsy and automated core biopsy for the diagnosis of gastrointestinal diseases in small animals. Vet. Radiol. and Ultrasound 34, 438-444.

Flückiger, M. and Arnold, P. (1986): Ultrasonic picture of intestinal invagination in the dog (in German). Kleintierpraxis 31, 379-380.

Grooters, A. M., Miyabayashi, T., Biller, D. S. and Merryman, J. (1994): Sonographic appearance of uremic gastropathy in four dogs. Vet. Radiol. and Ultrasound 35, 35-40.

Kantrowitz, B. M., Dimski, D., Swalec, K. and Biller, D. S. (1988): Ultrasonographic detection of jejunal intussusception and acute renal failure due to ethylene glycol toxicity in a dog. J. Am. Anim. Hosp. Assoc. 24, 697-700.

Kaser-Hotz, B., Hauser, B. and Arnold, P. (1996): Ultrasonographic findings in canine gastric neoplasia in 13 patients. Vet. Radiol. and Ultrasound 37, 51-56.

Lagalla, R., Caruso, G., Novara, V., Derchi, L. E. and Cardinale, A. E. (1994): Color Doppler ultrasonography in pediatric intussusceptions. J. Ultrasound Med. 13, 171-174.

Lamb, C. R. (1999): Recent developments in diagnostic imaging of the gastrointestinal tract of the dog and cat. Vet. Clin. North Am: Small Anim. Pract. 29, 307-342.

Lamb, C. R. and Simpson, K. W. (1995): Ultrasonographic findings in cholecystokinin-induced pancreatitis in dogs. Vet. Radiol. and Ultrasound 36, 139-145.

Manczur, F., Vörös, K., Vrabély, T., Wladár, S., Németh, T. and Fenyves, B. (1998): Sonographic diagnosis of intestinal obstruction in the dog. Acta Vet. Hung. 46, 35-45.

Mittelstaedt, C. A. (1992): Gastrointestinal Tract. In: Mittelstaedt, C. A. (ed.) General Ultrasound. Churchill Livingstone Inc. pp. 449-587.

Myers, N. C. and Penninck, D. G. (1994): Ultrasonographic diagnosis of gastrointestinal smooth muscle tumors in the dog. Vet. Radiol. and Ultrasound 35, 391-397.

Nyland, T. G., Mattoon, J. S. and Wisner, E. R. (1995): Ultrasonography of the pancreas. In: Nyland, T. G. and Mattoon, J. S. (eds) Veterinary Diagnostic Ultrasound. W. B. Saunders Co., Philadelphia. pp. 85-94. 
Penninck, D. G. (1995): Ultrasonography of the gastrointestinal tract. In: Nyland, T. G. and Mattoon, J. S. (eds) Veterinary Diagnostic Ultrasound. W. B. Saunders Co., Philadelphia. pp. $125-140$.

Penninck, D., Crystal, M. A., Matz, M. E. and Pearson, S. H. (1993): The technique of percutaneous ultrasound-guided fine-needle aspiration biopsy and automated core biopsy in small animal gastrointestinal diseases. Vet. Radiol. and Ultrasound 34, 433-436.

Penninck, D., Matz, M. and Tidwell, A. (1997): Ultrasonography of gastric ulceration in the dog. Vet. Radiol. and Ultrasound 38, 308-312.

Penninck, D. G., Moore, A. S. and Gliatto, J. (1998): Ultrasonography of canine gastric epithelial neoplasia. Vet. Radiol. and Ultrasound 39, 342-348.

Penninck, D. G., Nyland, T. G., Fisher, P. E. and Kerr, L. Y. (1989): Ultrasonography of the normal canine gastrointestinal tract. Vet. Radiol. 30, 272-276.

Penninck, D. G., Nyland, T. G., Kerr, L. Y. and Fisher, P. E. (1990): Ultrasonographic evaluation of gastrointestinal diseases in small animals. Vet. Radiol. 31, 134-141.

Rivers, B. J., Walter, P. A., Johnston, G. R., Feeney, D. A. and Hardy, R. M. (1997): Canine gastric neoplasia: Utility of ultrasonography in diagnosis. J. Am. Anim. Hosp. Assoc. 33, 144-155.

Saunders, H. M. (1991): Ultrasonography of the pancreas. Problems in Vet. Med. 3, 583-603.

Spohr, A., Koch, J. and Jensen, A. L. (1995): Ultrasonographic findings in a Basenji with immunoproliferative enteropathy. J. Small Anim. Pract. 36, 79-82.

Tidwell, A. S. and Penninck, D. G. (1992): Ultrasonography of gastrointestinal foreign bodies. Vet. Radiol. 33, 160-169.

Watson, D. E., Mahaffey, M. B. and Neuwirth, L. A. (1991): Ultrasonographic detection of duodenojejunal intussusception in a dog. J. Am. Anim. Hosp. Assoc. 27, 367-369. 